\title{
STUDY OF FACTORS INFLUENCING THE RISK AND THEIR RELATION TO CREDIBILITY THEORY
}

\author{
Ifaria Amélia Cabral and Jorge Afonso Garcia \\ Lisbon
}

\section{INTRODUCTION}

The study and analysis of the various factors influencing insurance risks constitutes an intricate and usually quite extensive problem. We have to consider on the one hand the nature and heterogeneity of the elements we have been able to measure, and on the other the problem of deciding-without knowing exactly what results to expect-on the types of analysis to carry out and the form in which to present the results.

These difficulties, essentially stemming from the fact that we cannot easily define "a priori" a measure of influence, can be overcome only by using highly sophisticated mathematical models. The researcher must define his objectives clearly if he is to avoid spending too much of his time in exploring such models.

Either for these reasons or for lack of our experience in this field we were led to the study of three models, presenting entirely different characteristics though based on the analysis and behaviour of mean value fluctuations, measured by their variances or by the least-squares method.

Our first model, described in II. I, associates the notion of influence with the notion of variance. It analyses in detail the alteration of the mean values variance, when what we refer to as a "margination" is executed in the parameter space, taking each of the parameters in turn. We start off by having $n$ distinct parameters, reducing them by one with each step.

As a complement of this method and allowing for an influence of residual character due to ignored or simply unknown factors, we tried to introduce a small correction to the usual credibility coefficients in order to provide for the explicit appearance of this residual influence. This type of influence is closely related to the existence of a tariff for the collective we are considering. 
The second model, described in II. 3, is fundamentally based on the least-squares method, and the way in which the influences are constructed and determined closely relates it to credibility theory.

The application of the resulting model seems simple and practical, although its theoretical study still needs a great deal of development, but unfortunately we were not able to carry it out in time for it to be incorporated in this paper.

The third model, briefly described in III. 2, is based on the $\chi^{2}$ test, giving it classical characteristics which lead to a laborious form of analysis and the determination of innumerable distribution functions (D.F.). For this reason the only purpose it served was that of testing the values obtained by the other two methods.

Finally, in III, we give numerical examples of the models we have described, comparing them and discussing their practical application.

\section{Description OF THE MODELS}

\section{Variance method}

Consider a collective $\theta$ composed of risks $\theta$ characterized by $n$ distinct parameters corresponding to $n$ factors, whose influence we wish to determine.

For simplicity suppose that all parameters assume positive integers

$$
\begin{aligned}
& \theta_{1}=I, 2,3, \ldots, k_{1} \\
& \vdots \\
& \theta_{2}=I, 2,3, \ldots, k_{2} \\
& \vdots \\
& 0_{n}=I, 2,3, \ldots, k_{n}
\end{aligned}
$$

We then have $\theta=\left(\theta_{1}, \theta_{2}, \ldots, \theta_{n}\right)$. The structure function $U(\theta)$ defined in the collective $\theta$ represents the D.F. of the risks $\theta$ in that collective.

Let $\mu_{i j k \ldots} \ldots$ and $p_{i j k \ldots}$ be respectively the mean value of the risk $\theta$ (in which $\theta_{1}=i, \theta_{2}=j, \theta_{3}=k, \ldots$ ) and the probability of randomly extracting that risk from the collective, that is the probability corresponding to the D.F. $U(\theta)$. 
We should note that knowing $U(\theta)$ and $\theta$ itself does not mean necessarily that $\theta$ is the real risk parameter, that is, $\theta$ merely represents the known vector corresponding to the factors being considered. Obviously for each risk there will be a more general unknown parameter of which $\theta$ is part. Thus, we can use for a certain risk the parameter $\left(\theta_{T}, \theta_{R}\right), \theta_{T}$ being the known part of the parameter and $\theta_{R}$ the unknown, corresponding to the ignored or unknown factors. In this case, the true structure function $U\left(\theta_{T}, \theta_{R}\right)$ will also be unknown. Thus, the values we will use correspond in a certain way to the marginal values $U\left(\theta_{T},.\right), \mu\left(\theta_{T},.\right)$ and $\left(\theta_{T},.\right)$.

Let us consider the marginal values corresponding to the margination carried out in the parameter space $T=\left\{\left(\theta_{1}, \theta_{2}, \ldots \theta_{n}\right)\right\}$ when one or more of these parameters are no longer considered.

Thus:

$$
\begin{aligned}
& \mu \cdot j k \ldots=\frac{\sum_{i=1}^{k_{1}} \mu_{i j k \ldots} \ldots p_{i j k \ldots} \ldots}{\sum_{i=1}^{k_{2}} p_{i j k \ldots} \ldots} ; p \cdot j k \ldots=\sum_{i=1}^{k_{1}} p_{i j k \ldots} \ldots \\
& \mu . . k \ldots=\frac{\sum_{i .1}^{\Sigma} \mu_{i j k} \ldots p_{i j k \ldots}}{\sum_{i, j} p_{i j k \ldots} \ldots} ; p . . k \ldots=\sum_{i, 1} p_{i j k \ldots} \ldots
\end{aligned}
$$

Considering the variances of $\mu\left(\theta_{1}, \theta_{2}, \ldots, \theta_{n}\right), \mu\left(., \theta_{2}, \ldots, \theta_{n}\right)$, $\mu\left(., ., \theta_{3}, \ldots, \theta_{n}\right)$, etc., and their respective differences

$$
\begin{aligned}
& V=\sum_{i, i, k, \ldots} \mu_{i j k}^{2} \ldots p_{i j k \ldots}-\mu^{2} \quad \text { with } \mu=E_{T}[\mu(\theta)] \\
& V_{1}=\sum_{1, k, \ldots}^{\Sigma} \mu_{\cdot j k \ldots}^{2} \ldots p_{\cdot j k} \ldots-\mu^{2} \quad \text { (margining in } \theta_{1} \text { ) } \\
& V_{2}=\sum_{i, k, \ldots} \mu_{i \cdot k}^{2} \ldots p_{i \cdot k} \ldots-\mu^{2} \quad \text { (margining in } \theta_{2} \text { ) } \\
& V_{12}=\sum_{k \ldots} \mu_{*}^{2} \ldots \ldots p_{*} \ldots \ldots-\mu^{2} \\
& I_{1}=V-V_{1}, I_{2}=V-V_{2}, \ldots, I_{12}=V-V_{12}, \ldots
\end{aligned}
$$


We can see that the "operation" of margination, as it levels the mean values, nearly always causes a lowering of the variance, which can be seen by the following theorem.

\section{Theorem I}

Considering $\theta=\left(\theta_{1}, \theta_{2}, \ldots, \theta_{n}\right)$ and $\theta^{\prime}=\left(\theta_{2}, \ldots, \theta_{n}\right)$ (without loss of generality) the following inequality is always true:

$$
\operatorname{Var}\left[\mu\left(\theta^{\prime}\right)\right] \leqslant \operatorname{Var}[\mu(\theta)]
$$

Proof:

For simplicity we will only use two parameters: $\theta_{1}$ and $\theta_{2}$.

Thus

$$
\theta=\left(\theta_{1}, \theta_{2}\right) ; \quad \theta^{\prime}=\theta_{2}
$$

We then have

and

$$
\operatorname{Var}[\mu(\theta)]=\sum_{i,} \mu_{i j}^{2} p_{i j}-\mu^{2}=V
$$

$$
\begin{gathered}
\operatorname{Var}\left[\mu\left(\theta^{\prime}\right)\right]=\sum_{j} \mu_{. j}^{2} p_{. j}-\mu^{2}=V_{1} \\
I_{1}=V-V_{1}=\sum_{i,} \mu_{i j}^{2} p_{i j}-\sum_{j} \mu_{. j}^{2} p_{. j}
\end{gathered}
$$

making $\mu_{i j}=\mu \cdot j+\alpha_{i j}$ we have

which implies

$$
\mu \cdot j=\mu \cdot j+\frac{\sum \alpha_{i j} p_{i j}}{p \cdot j}
$$

In this way

$$
\sum_{i} \alpha_{i j} p_{i j} \equiv 0 \quad \forall j
$$

$I_{1}=\sum_{i}\left(\mu_{. j}^{2} p_{. j}+\sum_{i} \alpha_{i j}^{2} p_{i j}+2 \mu_{. j} \sum_{i} \alpha_{i j} p_{i j}-\mu_{. j}^{2} p_{. j}\right)=\sum_{i, j} \alpha_{i j}^{2} p_{i j}$

\section{Corollary I}

We see that the values of $I_{1}, I_{2}, \ldots, I_{12}, \ldots$ defined previously never have negative values.

\section{Corollary 2}

We can easily verify that $I_{1}=0$ if and only if $\alpha_{i j} \equiv 0$ that is $\mu_{i j}=\mu \cdot j$ for all $j$. 
This corollary gives us a first approximation to the influence concept, since $\mu_{i j}=k \cdot j$ implies that, at least considering the mean values, $\theta_{1}$ has no influence.

A second approximation to this concept will be given by the following notion of independence, defined only for mean values.

\section{Definition of independence}

We can say that $\theta_{1}$ and $\theta_{2}$ have independent influences on the risk, if and only if the variation of $\mu_{i j}$ with $i$ is independent of the value of $j$. We should note that this notion is a particular case of the true notion of independence, which should be set out in the same manner, by using the D.F. of the total amount of claims during a certain period.

From this definition we arrive at the following theorem:

\section{Theorem 2}

If $\theta_{1}$ and $\theta_{2}$ are independent through their distribution in the collective $\Theta$ and if they have independent influences, then

$$
I_{12}=I_{1}+I_{2}
$$

Proof:

By the definition of independence in relation to the D.F. $U(\theta)$, we can write the following equality:

$$
p_{i j k l}=\frac{p_{\cdot j k l} p_{i \cdot k l}}{p \cdot \cdot k l}
$$

By the hypothesis of independent influences on the risk, we can also write

$$
\mu_{i j k l}=\mu_{1 j k l}+\alpha_{i k l} \quad \forall j
$$

We then have

$$
\mu \cdot j k l=\mu_{1 j k l}+\frac{\sum \alpha_{i k l} p_{i j k l}}{p \cdot j k l}
$$

or taking

$$
\begin{aligned}
& \frac{\sum_{i} \alpha_{i k l} p_{i j k l}}{p \cdot j k l}=\alpha \cdot k l(j) \\
& \mu \cdot j k l=\mu_{1 j k l}+\alpha \cdot k l(j)
\end{aligned}
$$


On the other hand, we have

$$
i_{i \cdot k l}=\mu_{1 \cdot k l}+\alpha_{i k l}
$$

and

$$
\mu . . k l=\mu_{1 \cdot k l}+\frac{\sum \alpha_{i k l} p_{i j k l}}{p \cdot . k l}=\mu_{1 \cdot k l}+\alpha \cdot k l
$$

in which

$$
\alpha \cdot k l=\frac{\sum \alpha \cdot k l(j) p \cdot j k l}{p \cdot \cdot k l}
$$

Since .

$$
\begin{aligned}
I_{1}+I_{2}-I_{12}=V-V_{1}-V_{9} & +V_{12}= \\
& =\sum_{k l} \alpha_{. k l}^{2} p_{. . k l}-\sum_{j k l} \alpha_{. k l}^{2}(j) p_{. j k l}
\end{aligned}
$$

and

$$
\alpha \cdot k l(j)=\frac{\sum \alpha_{i k l} p_{i j k l}}{p \cdot j k l}=\frac{\sum_{i} \alpha_{i k l} p_{\cdot j k l} p_{i \cdot k l}}{p \cdot j k l p \cdot \cdot k l}=\frac{\sum_{i} \alpha_{i k l} p_{i} \cdot k l}{p \cdot \cdot k l}=x \cdot k l
$$

We conclude immediately that

$$
I_{12}=I_{1}+I_{2}
$$

We should note that the inverse property of theorem of 2 is not always true.

From the previous theorem we can conclude that

$$
I_{i j} \ldots \leqslant V
$$

In summary, the values $I$ have the following properties:

$\mathrm{I}-I(\theta) \geqslant 0$

$2-I\left(\theta_{1} \cdot \theta_{2}\right)=I\left(\theta_{1}\right)+I\left(\theta_{2}\right)$ if $\theta_{1}$ and $\theta_{2}$

are independent, that is, if they are uncorrelated in the ways described above.

The operation $\theta_{1} \circ \theta_{2}$ corresponds to the "union" of influences and not to its "intersection" as one could be led to believe, as it is a global influence of $\theta_{1}$ and $\theta_{2}$. 
By the properties $I$ and 2 we can treat the value $I(\theta)$ as the measure of the influence of the parameter on the risk.

Comparing the second property with the union of events defined in the probability space, characterized by

$$
\begin{aligned}
& P\left(A_{1} U A_{2} U \ldots U A_{n}\right)=P\left(A_{1}\right)+P\left(A_{2}\right)+\ldots+P\left(A_{n}\right)- \\
& -P\left(A_{1} \cap A_{2}\right)-\ldots-P\left(A_{n-1} \cap A_{n}\right)+\ldots+ \\
& +(-\mathrm{I})^{n+1} P\left(A_{1} \cap A_{2} \cap \ldots \cap A_{n}\right),
\end{aligned}
$$

it is possible to generalize that property giving it a similar form. In order to make the comparison more evident we can still write

$$
P\left(A_{i} \cap A_{\jmath}\right)=C P\left(A_{i}, A_{\jmath}\right)
$$

if we consider the probability of the "intersection" of events as the "coprobability" between $A_{i}$ and $A_{f}$.

\section{Theorem 3}

Representing $C I_{12} \ldots k$ by the following expression:

$$
\begin{aligned}
C I_{12 \ldots k} & =V-V_{1}-\ldots- \\
& -V_{k}+V_{12}+\ldots+V_{k-1 k}+\ldots(-\mathrm{I})^{k} V_{12} \ldots k
\end{aligned}
$$

$k$ being the number of the parameters considered, we can write:

$$
\begin{aligned}
I\left(\theta_{1}, \theta_{2}, \ldots, \theta_{n}\right) & =I_{1}+I_{2}+\ldots+I_{n}- \\
& -C I_{12}-\ldots-C I_{n-1 n}+\ldots+(-I)^{n+1} C I_{12} \ldots n
\end{aligned}
$$

Proof:

For simplicity, consider only three parameters: $\theta_{1}, 0_{2}$ and $\theta_{3}$.

We have,

$$
\begin{aligned}
& I_{1}=V-V_{1} \\
& I_{2}=V-V_{2} \\
& I_{3}=V-V_{3} \\
& C I_{12}=V-V_{1}-V_{2}+V_{12} \\
& C I_{13}=V-V_{1}-V_{3}+V_{13} \\
& C I_{23}=V-V_{2}-V_{3}+V_{23} \\
& C I_{123}=V-V_{1}-V_{2}-V_{3}+V_{12}+V_{13}+V_{23}-V_{123} \\
& I_{1}+I_{2}+I_{3}-C I_{12}-C I_{13}-C I_{23}+C I_{123}=V-V_{123} .
\end{aligned}
$$

As we have only three parameters, $V_{123}=0$.

Thus the theorem is proved. 
By this theorem and comparing the coprobabilities with the $C I$ we can say that they are in a certain way the measure of the coinfluence.

We could also show by a laborious set of calculations that when $I_{1}, I_{2}, \ldots, I_{n}$ have a variance structure, $C I_{12}, \ldots, C I_{n-1 n}$ have a generalized covariance structure.

If we consider the following diagram:

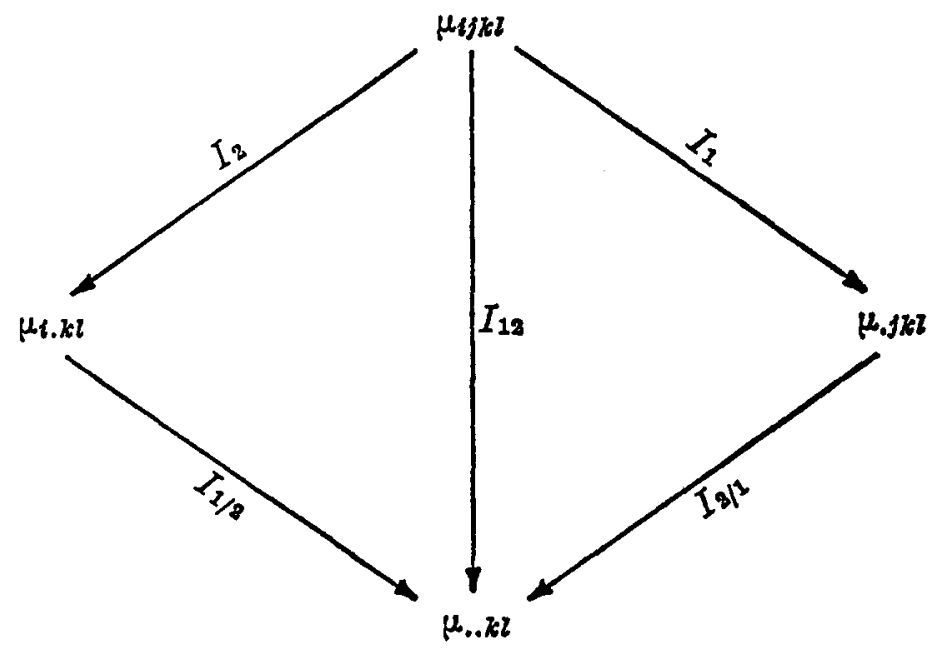

we can establish the following relations, easily verified by the previous theorems:

$$
I_{12}=I_{1}+I_{2 / 1}=I_{2}+I_{1 / 2}
$$

In a general way $I_{12} \leqslant I_{1}+I_{2}$ the equality being verified if and only if $I_{2}=I_{2 / 1}$ and $I_{1}=I_{1 / 2}$ that is, in the case of the factors $\theta_{1}$ and $\theta_{2}$ having independent influences.

We note that it is not easy to establish for $I(\theta)$ a measure space, similar to the probability spaces or to other spaces defined in the sense of measure theory.

At this point it is important to realize that $C I$ can assume negative values. The measure $I$ resembles the notion of a force not only in its nature but also in its effect.

The influence of a certain factor can be considered a type of a potential force which, isolated or in conjunction with others, will bring about a claim. 
Although $I\left(\theta_{i}\right)$ defines in a certain way the measure of influence of the parameter $\theta_{i}$, we are interested in a global measure rather than considering the influence on its own.

Thus, we are interested in the "measure" of the effect or the contribution of the parameter, in conjunction with all other influences. In this case, we are obliged to consider the coinfluences, which could be as important as, or more important than, the influences themselves. How then should we proceed?

It seems that an input of the $C I$ proportional to the influences of each parameter could solve the problem. However, we have to admit that such a procedure involves some risks. For example, an isolated parameter could appear to have a weak influence and contribute a small value for $I$, and with its association with other parameters, specially for certain particular values, could have a very strong influence. In this case, the method we have followed would fail completely. We think that common sense, aided by discussion with the manager responsible for the class of business being considered, should ensure that no serious mistakes are made.

2. The existence of a residual influence and its relation to the credibility premium

There should be a difference between the value of $I\left(\theta_{1}, \theta_{2}, \ldots, \theta_{n}\right)$ and the true value of $\operatorname{Var}[\mu(\theta)]$ representing the global influence of all factors.

In effect, working with the marginal value $\left(\theta_{T}, \cdot\right)$ instead of $\left(\theta_{T}, \theta_{R}\right)$ will give in the general case $I\left(\theta_{1}, \theta_{2}, \ldots, \theta_{n}\right)<\operatorname{Var}[\mu(\theta)]$, a direct consequence of theorem $I$.

As it is relatively simple to estimate $\operatorname{Var}[\mu(\theta)]$ for the collective and as we calculate the value of $I$, we would be left with the difference $I\left(\theta_{R}\right)$ which we will call the residual influence.

Thus, the following equality will hold

$$
\operatorname{Var}[\mu(\theta)]=I\left(\theta_{1}, \theta_{2}, \ldots, \theta_{n}\right)+I\left(\theta_{R}\right) .
$$

When the factors $\theta_{1}, \theta_{2}, \ldots, \theta_{n}$ are those considered by the tariff we call $I\left(\theta_{1}, \theta_{2}, \ldots, \theta_{n}\right)$ the influence of the tariff, $I\left(\theta_{T}\right)$.

Thus, the previous equality will be written as follows:

$$
\operatorname{Var}[\mu(\theta)]=I\left(\theta_{T}\right)+I\left(\theta_{R}\right)
$$


We can also see that the more factors (among those having an influence on the risk) that we eliminate from the tariff, the more significant the residual influence will be.

This very often leads to highly, heterogeneous classes of risks, and eventually an unsuitable tariff structure. Thus, in general the tariff premium is nothing more than an indicator of the characteristics of risks to which it is applied, so that it is often necessary to readapt the risk premium by using a credibility premium. It can still happen that because of the choice of parameters, the risk is placed in a tariff class different from that in which it would be placed if the intrinsic values were used. The previous considerations lead us to believe in the need to calculate the credibility premium, modifying it by the value of the residual influence.

Once again let us consider the collective, over which we suppose a tariff is defined by parameters $\theta_{T}$. Still considering a set of unknown parameters denoted by $\theta_{R}$, each risk $\theta$ would then be characterized by the pair $\left(\theta_{T}, \theta_{R}\right)$.

As we stated previously, $U\left(\theta_{T}, \theta_{R}\right)$ is unknown, but we do know its marginal $U\left(\theta_{T},.\right)$ characterizing the distribution of the risks in the collective tariff classes.

In the same way

$$
\mu\left(\theta_{T}, \theta_{R}\right)=\int x d G^{\left(\theta_{T}, 0_{R}\right)}(x)
$$

is unknown.

Nevertheless the value

$$
\mu\left(\theta_{T}, .\right)=\int x d G^{\left(\theta_{T},\right)}(x)=\frac{\int_{R} \mu\left(\theta_{T}, \theta_{R}\right) d U\left(0_{T}, \theta_{R}\right)}{\int_{R} d U\left(\theta_{T}, \theta_{R}\right)}
$$

is known, $G^{\left(\theta^{\prime} \cdot\right)}(x)$ being the D.F. of the total amount of claims corresponding to the tarif class $\theta_{T}$.

In the same manner we have

$$
\begin{aligned}
\mu & =E[\mu(\theta)]=\int_{T \times R} \mu\left(\theta_{T}, \theta_{R}\right) d U\left(\theta_{T}, \theta_{R}\right) \\
& =\int_{T} \mu\left(\theta_{T}, .\right) d U\left(\theta_{T}, .\right) \\
& =E\left[\mu\left(\theta_{T}, .\right)\right] .
\end{aligned}
$$


Now we shall deduce certain expressions which will be needed in the calculation of the credibility coefficients.

I.

$$
E_{T \times R}\left[\mu\left(\theta_{T}, \theta_{R}\right) \times \mu\left(\theta_{T}, .\right)\right]=E_{T}\left[\mu^{2}\left(\theta_{T}, .\right)\right],
$$

since

$$
\begin{aligned}
& \int_{T \times R} \mu\left(\theta_{T}, \theta_{R}\right) \times \mu\left(\theta_{T}, .\right) d U\left(\theta_{T}, \theta_{R}\right)= \\
& \quad=\int_{T} \mu\left(\theta_{T}, .\right) \int_{R} \mu\left(\theta_{T}, \theta_{R}\right) d U\left(\theta_{T}, \theta_{R}\right)=\int_{T} \mu^{2}\left(\theta_{T}, .\right) d U\left(\theta_{T}, .\right)
\end{aligned}
$$

2.

$$
E_{S \times T \times R}\left[\bar{S} \times \mu\left(\theta_{T}, \theta_{R}\right)\right]=E_{T \times R}\left[\mu^{2}\left(\theta_{T}, \theta_{R}\right)\right]
$$

where

$$
\bar{S}=\frac{S_{1}+S_{2}+\ldots+S_{n}}{n},
$$

$S_{i}$ being the global amount of the observed claims of each risk during the period $i$.

Proof:

$$
\begin{aligned}
E_{S \times T}<R & {\left[\bar{S} \times \mu\left(\theta_{T}, \theta_{R}\right)\right] } \\
& \left.=\int_{s \times T \times R} \bar{S} \times \mu\left(\theta_{T}, \theta_{R}\right) d W\left[\left(S_{1}, S_{2}, \ldots, S_{n}\right) /\left(\theta_{T}, \theta_{R}\right)\right] d U\left(\theta_{T}, \theta_{R}\right)^{1}\right) \\
& \left.=\int_{s \times T \times R} \bar{S} \times \mu\left(\theta_{T}, \theta_{R}\right) d G^{\left(\theta_{T}, \theta_{R}\right)}\left(S_{1}\right) \ldots d G^{\left(\theta_{T}, \theta_{R}\right)}\left(S_{n}\right) d U\left(\theta_{T}, \theta_{R}\right)^{1}\right) \\
& =\int_{T \times R} \mu\left(\theta_{T}, \theta_{R}\right)\left[\iint_{S} \bar{S} d G^{\left(\theta_{T}, \theta_{R}\right)}\left(S_{1}\right) \ldots d G^{\left(\theta_{T}, \theta_{R}\right)}\left(S_{n}\right)\right] d U\left(\theta_{T}, \theta_{R}\right) \\
& =\int_{T \times R} \mu\left(\theta_{T}, \theta_{R}\right) E\left[\bar{S} /\left(\theta_{T}, \theta_{R}\right)\right] d U\left(\theta_{T}, \theta_{R}\right) \\
& =\int_{T \times R} \mu^{2}\left(\theta_{T}, \theta_{R}\right) d U\left(\theta_{T}, \theta_{R}\right) \\
& =E_{T \times R}\left[\mu^{2}\left(\theta_{T}, \theta_{R}\right)\right] .
\end{aligned}
$$

We note that

$$
E\left[\bar{S} /\left(\theta_{T}, \theta_{R}\right)\right]=E\left[S_{i} /\left(\theta_{T}, \theta_{R}\right)\right]=\mu\left(\theta_{T}, \theta_{R}\right)
$$

1) The relation is justified by Bayes's theorem and the assumed indepence of $S_{1}, S_{2}, \ldots, S_{n}$. 
3.

$$
E_{S \times T \times R}\left[\bar{S} \times \mu\left(\theta_{T}, \cdot\right)\right]=E_{T}\left[\mu^{2}\left(\theta_{T}, .\right)\right]
$$

From 2, we have

$$
\begin{aligned}
& E_{S \times T \times R}\left[\bar{S} \times \mu\left(\theta_{T}, .\right)\right]=\int_{T \times R} \mu\left(\theta_{T}, .\right) \times \mu\left(\theta_{T}, \theta_{R}\right) d U\left(\theta_{T}, \theta_{R}\right) \\
& =\int_{T} \mu\left(\theta_{T}, .\right) \int_{R} \mu\left(\theta_{T}, \theta_{R}\right) d U\left(\theta_{T}, \theta_{R}\right) \\
& =\int_{T} \mu^{2}\left(\theta_{T}, .\right) d U\left(\theta_{T}, .\right)=E_{T}\left[\mu^{2}\left(\theta_{T}, .\right)\right]
\end{aligned}
$$

4.

$$
E_{T \times R}\left[\mu\left(\theta_{T}, \theta_{R}\right) \times \mu\left(\theta_{T}, .\right)\right]=E_{S \times T \times R}\left[\bar{S} \times \mu\left(\theta_{T}, .\right)\right]
$$

\section{Credibility Premium}

Linearisation of the expected value part.

The fundamental problem resides in the determination of

$$
E_{R}\left[\mu\left(\theta_{T}, \theta_{R}\right) / S_{1}, S_{2}, \ldots, S_{n}\right]
$$

knowing the value $\mu\left(\theta_{T},.\right)$.

We will try to approximate to that value by the usual method of minimizing the variance in the collective. Considering the equality

$$
E_{R}\left[\mu\left(0_{T}, \theta_{R}\right) / S_{1}, S_{2}, \ldots, S_{n}\right]=a+b \mu\left(\theta_{T}, .\right)+c \bar{S}
$$

where the constants $a, b$ and $c$ are determined by minimization of the following expression

$E_{S \times T}\left[\left\{E_{R}\left[\mu\left(\theta_{T}, \theta_{R}\right) / S_{1}, S_{2}, \ldots, S_{n}\right]-\left[a+b \mu\left(\theta_{T}, .\right)+c \bar{S}\right]\right\}^{2}\right]$

We followed two criteria to determine the values of these constants.

Following Prof. Bühlmann we can easily see that minimizing the expression $(A)$ is equivalent to minimizing

$$
\begin{aligned}
& E_{S \times T \times R}\left[\left\{\mu\left(\theta_{T}, \theta_{R}\right)-\left[a+b \mu\left(\theta_{T}, .\right)+c \bar{S}\right]\right\}^{2}\right] \\
= & E_{S \times T \times R}\left[\left\{(b+c)\left[\mu\left(\theta_{T}, \theta_{R}\right)-\mu\left(\theta_{T}, .\right)\right]+\left[c\left[\mu\left(\theta_{T}, .\right)-\bar{S}\right]\right]+\right.\right. \\
+ & {\left.\left.\left[(\mathrm{I}-b-c) \mu\left(\theta_{T}, \theta_{R}\right)-a\right]\right\}^{2}\right] }
\end{aligned}
$$

Putting

$$
\left\{\begin{array}{l}
\alpha=E\left[\left\{\mu\left(\theta_{T}, \theta_{R}\right)-\mu\left(\theta_{T}, .\right)\right\}^{2}\right] \\
\beta=E\left[\left\{\mu\left(\theta_{T}, .\right)-\bar{S}\right\}^{2}\right] \\
\gamma=E\left[\mu^{2}\left(\theta_{T}, \theta_{R}\right)-\mu^{2}\left(\theta_{T}, .\right)\right]
\end{array}\right.
$$


and developing $(B)$ we obtain the following expression $f(b, c)=(b+c)^{2} \alpha+c^{2} \beta+(\mathrm{I}-b-c)^{2} \operatorname{var}\left[\mu\left(\theta_{T}, \theta_{R}\right)\right]-$ $-2\left(b^{2}+c^{2}+2 b c-b\right) \gamma$

Taking the partial derivatives of the function $f(b, c)$ and resolving the system

$$
\left\{\begin{array}{l}
\frac{\partial f}{\partial b}=0 \\
\frac{\partial f}{\partial c}=0
\end{array}\right.
$$

we obtain the following values

$$
\begin{aligned}
& c=\frac{Y}{\beta} \\
& b=I-\frac{\gamma}{\beta} \longrightarrow a=0
\end{aligned}
$$

2. Starting on the L.H.S. of the expression defined in (B), squaring it out and taking derivatives, we have:

$$
\left\{\begin{array}{l}
a-E\left[\mu\left(\theta_{T}, \theta_{R}\right)\right]+b E\left[\mu\left(\theta_{T}, .\right)\right]+c E(\bar{S})=0 \\
b E\left[\mu^{2}\left(\theta_{T}, .\right)\right]-E\left[\mu\left(\theta_{T}, \theta_{R}\right) \times \mu\left(\theta_{T}, .\right)\right]+a E\left[\mu\left(\theta_{T}, .\right)\right]+ \\
\quad+c E\left[\mu\left(\theta_{T}, .\right) \times \bar{S}\right]=0 \\
c E\left(\bar{S}^{2}\right)-E\left[\mu\left(\theta_{T}, \theta_{R}\right) \times \bar{S}\right]+a E(\bar{S})+b E\left[\mu\left(\theta_{T}, .\right) \times \bar{S}\right]=0
\end{array}\right.
$$

From these equations we can obtain:

$$
\begin{aligned}
a & =(I-b-c) \mu=0 \\
b & =I-\frac{E\left[\mu^{2}\left(\theta_{T}, \theta_{R}\right)\right]-E\left[\mu^{2}\left(\theta_{T}, \cdot\right)\right]}{E\left[\left\{\mu\left(\theta_{T}, .\right)-\bar{S}\right\}^{2}\right]} \\
c & =\frac{E\left[\mu^{2}\left(\theta_{T}, \theta_{R}\right)\right]-E\left[\mu^{2}\left(\theta_{T}, .\right)\right]}{E\left[\left\{\mu\left(\theta_{T}, .\right)-\bar{S}\right\}^{2}\right]}
\end{aligned}
$$

Finally we will have for the expected value part of the credibility premium the following linearisation

$$
E\left[\mu\left(\theta_{T}, \theta_{R}\right) / S_{1}, \ldots, S_{n}\right]=(I-c) \mu\left(\theta_{T}, . .\right)+c \bar{S}
$$


If we compare this expression to that normally considered in credibility theory, that is

$$
E\left[\mu(\theta) / S_{1}, \ldots, S_{n}\right]=(\mathrm{I}-b) \mu+b \bar{S}
$$

we note that they are of the same form. On the other hand, comparing the credibility coefficients $c$ and $b$, in which

$$
\begin{gathered}
c=\frac{\operatorname{var}\left[\mu\left(\theta_{T}, \theta_{R}\right)\right]-\operatorname{var}\left[\mu\left(\theta_{T}, \cdot\right)\right]}{\operatorname{var}(\bar{S})-\operatorname{var}\left[\mu\left(\theta_{T}, \cdot\right)\right]} \\
b=\frac{\operatorname{var}\left[\mu\left(\theta_{T}, \theta_{R}\right)\right]}{\operatorname{var}(\bar{S})}
\end{gathered}
$$

we can conclude that the expression (2) is a particular case of (I) if no tariff is considered over the collective.

We can easily see that $c \leqslant b$.

Taking $b=\frac{x}{y}$ and $c=\frac{x-z}{y-z}$ where $x, y, z>0, x<y$ and $z<y$ we have:

$$
\begin{aligned}
c-b & =\frac{x-z}{y-z}-\frac{x}{y}=\frac{x y-y z-x y+x z}{y(y-z)} \\
& =\frac{z(x-y)}{y(y-z)}<0
\end{aligned}
$$

If we use the value of $b$ obtained by the expression (2) for all the collective independently of the tariff class, we verify the following theorem.

\section{Theorem 4}

If we consider the collective $\Theta$ partitioned into well defined classes of risk, the following inequality holds:

$$
E\left[\left\{\mu\left(\theta_{T}, \theta_{R}\right)-(I-c) \mu\left(\theta_{T}, .\right)-c \bar{S}\right\}^{2}\right]<
$$

$$
<E\left[\left\{\mu\left(\theta_{T}, \theta_{R}\right)-(I-b) \mu-b \bar{S}\right\}^{2}\right]
$$


Proof:

$$
\begin{aligned}
(4)= & E\left[\left\{\left(\mu\left(\theta_{T}, \theta_{R}\right)-\mu\right)+b(\mu-\bar{S})\right\}^{2}\right] \\
= & \operatorname{var}\left[\mu\left(\theta_{T}, \theta_{R}\right)\right]+b^{2} \operatorname{var}(\bar{S})+2 b \mu E\left[\mu\left(\theta_{T}, \theta_{R}\right)\right]- \\
& \quad-2 b E\left[\mu\left(\theta_{T}, \theta_{R}\right) \times \bar{S}\right]-2 b \mu^{2}+2 b \mu E(\overline{S)} \\
= & \operatorname{var}\left[\mu\left(\theta_{T}, \theta_{R}\right)\right]+b^{2} \operatorname{var}(\bar{S})+2 b \mu^{2}- \\
& -2 b E\left[\mu^{2}\left(\theta_{T}, \theta_{R}\right)\right]-2 b \mu^{2}+2 b \mu^{2} \\
= & \operatorname{var}\left[\mu\left(\theta_{T}, \theta_{R}\right)\right]+\frac{\operatorname{var}^{2} \mu\left(\theta_{T}, \theta_{R}\right)}{\operatorname{var}(\bar{S})}-2 \frac{\operatorname{var}\left[\mu\left(\theta_{T}, \theta_{R}\right)\right]}{\operatorname{var}(\bar{S})} \\
= & \operatorname{var}\left[\mu\left(\theta_{T}, \theta_{R}\right)\right] \times(\mathrm{I}-b) .
\end{aligned}
$$

On the other hand

$$
\begin{aligned}
(3)= & E\left[\left\{\left(\mu\left(\theta_{T}, \theta_{R}\right)-\mu\left(\theta_{T}, .\right)\right)+c\left(\mu\left(\theta_{T}, .\right)-\bar{S}\right)\right\}^{2}\right] \\
= & \operatorname{var}\left[\mu\left(\theta_{T}, \theta_{R}\right)\right]-\operatorname{var}\left[\mu\left(\theta_{T}, .\right)\right]+c^{2}[\operatorname{var}(\bar{S})- \\
& \left.-\operatorname{var}\left[\mu\left(\theta_{T}, .\right)\right]\right]+2 c E\left[\mu^{2}\left(\theta_{T}, .\right)-\mu^{2}\left(\theta_{T}, \theta_{R}\right)-\right. \\
= & \operatorname{var}\left[\mu\left(\theta_{T}, \theta_{R}\right)\right]-\operatorname{var}\left[\mu\left(\theta_{T}, .\right)\right]- \\
& \quad-\frac{\left\{\operatorname{var}\left[\mu\left(\theta_{T}, \theta_{R}\right)\right]-\operatorname{var}\left[\mu\left(\theta_{T}, .\right)\right]\right\}^{2}}{\operatorname{var}(\bar{S})-\operatorname{var}\left[\mu\left(\theta_{T}, .\right)\right]} \\
= & \left\{\operatorname{var}\left[\mu\left(\theta_{T}, \theta_{R}\right)\right]-\operatorname{var}\left[\mu\left(\theta_{T}, .\right)\right]\right\} \times(\mathrm{I}-c)
\end{aligned}
$$

Subtracting the two expressions

$$
\begin{aligned}
(4)-(3)=\frac{\operatorname{var}\left[\mu\left(\theta_{T}, .\right)\right]}{\left\{\operatorname{var}(\bar{S})-\operatorname{var}\left[\mu\left(\theta_{T}, .\right)\right]\right\}} & \operatorname{var}(\bar{S}) \\
\times & \left\{\operatorname{var}\left[\mu\left(\theta_{T}, \theta_{R}\right)\right]-\operatorname{var}(\bar{S})\right\}^{2} .
\end{aligned}
$$

As the numerator and the denominator are positive we conclude that

$$
\text { (4) }>(3)
$$

\section{Credibility influences methoul}

Let $\theta_{1}$ and $\theta_{2}$ be two parameters by which we want to determine the tariff for a certain risk. We can assume, without loss of generality, that $\mu\left(\theta_{1},.\right)<\mu\left(., \theta_{2}\right)$.

With similar reasoning as used in the construction of the credibility premium we can imagine two insurers $A$ and $B$ with the following philosophies: 
- A, the more optimistic, assumes that $\theta_{1}$ is the parameter with the greater influence and uses for the net premium the mean value $\mu\left(\theta_{1},.\right)$.

- $B$, the more pessimistic, assumes $\theta_{2}$ to be the more influential parameter and uses $\mu\left(., \theta_{2}\right)$ as his net premium.

If we imagine a further insurer $C$, without such extrame positions, he will attribute the intermediate value $\mu=\alpha_{1} \mu\left(\theta_{1},.\right)+\alpha_{2} \mu\left(., \theta_{2}\right)$ to the risk $\mu\left(\theta_{1}, \theta_{2}\right)$.

We are assuming that all of them ignore $\mu\left(\theta_{1}, \theta_{2}\right)$. We believe that if no other information is available, $\mathrm{C}$ will use intuitively, $\alpha_{1}=\alpha_{2}=0.5$.

If he thinks that $\theta_{1}$ has more influence than $\theta_{2}$ he will naturally use $\alpha_{1}>\alpha_{2}$ maintaining the sum $\alpha_{1}+\alpha_{2}=I$.

All in all, $\alpha_{1}$ and $\alpha_{2}$ represent the credibility attributed by $C$ to the factors, or better still, to each of their influences.

It seems that this philosophy can be generalized to all factors in order to obtain the desired measure of influence.

Consider $n$ parametrised factors $\theta_{1}, \theta_{2}, \ldots, \theta_{n}$ and assume that the marginal mean values $\mu_{1}=\mu\left(\theta_{1}, \ldots, \ldots\right), \mu_{2}=\mu\left(., \theta_{2}, \ldots, \ldots\right)$, etc. are known. The problem we wish to solve consists in approximating the unknown value $\mu\left(\theta_{T}, \theta_{R}\right)$ by the linear combination $\alpha_{1} \mu_{1}+\alpha_{2} \mu_{2}+\ldots+\alpha_{n} \mu_{n}$. Using the least-squares method normally applied in credibility theory we can determine $\alpha_{1}, \alpha_{2}, \ldots, \alpha_{n}$ by minimizing the expression

$$
E\left[\left\{\mu\left(\theta_{T}, \theta_{R}\right)-\left(\mu_{1} \alpha_{1}+\mu_{2} \alpha_{2}+\ldots+\mu_{n} \alpha_{n}\right)\right\}^{2}\right]
$$

Squaring out this expression we obtain:

$$
\begin{aligned}
E\left[\mu^{2}\left(\theta_{T}, \theta_{R}\right)\right]+\alpha_{1}^{2} E\left(\mu_{1}^{2}\right)+\ldots+\alpha_{n}^{2} E\left(\mu_{n}^{2}\right)- \\
-2 \alpha_{1} E\left[\mu_{1} \times \mu\left(\theta_{T}, \theta_{R}\right)\right]-\ldots-2 \alpha_{n} E\left[\mu_{n} \times \mu\left(\theta_{T}, \theta_{R}\right)\right]+ \\
+2 \sum_{i \neq j} \alpha_{i} \alpha_{j} E\left(\mu_{i} \times \mu_{j}\right)
\end{aligned}
$$

Taking derivatives in the other $\alpha_{1}, \alpha_{2}, \ldots, \alpha_{n}$, dividing by two and equating to zero, we have:

$$
\left\{\begin{array}{l}
\alpha_{1} E\left(\mu_{1}^{2}\right)-E\left(\mu_{1}^{2}\right)+\alpha_{2} E\left(\mu_{1} \times \mu_{2}\right)+\ldots+\alpha_{n} E\left(\mu_{1} \times \mu_{n}\right)=0 \\
\alpha_{2} E\left(\mu_{2}^{2}\right)-E\left(\mu_{2}^{2}\right)+\alpha_{1} E\left(\mu_{2} \times \mu_{1}\right)+\ldots+\alpha_{n} E\left(\mu_{2} \times \mu_{n}\right)=0 \\
\cdot \\
\cdot \\
\alpha_{n} E\left(\mu_{n}^{2}\right)-E\left(\mu_{n}^{2}\right)+\alpha_{1} E\left(\mu_{n} \times \mu_{1}\right)+\ldots+\alpha_{n-1} E\left(\mu_{n-1} \times \mu_{n}\right)=0
\end{array}\right.
$$


Writing $E_{i j}$ for $E\left[\mu_{i}, \times \mu \cdot j\right]$ the system becomes:

$$
\left[\begin{array}{cccc}
E_{11} & E_{12} & \ldots & E_{1 n} \\
E_{21} & E_{22} & \ldots & E_{2 n} \\
\cdot & & & \\
\cdot & & & \\
\cdot & & & \\
E_{n 1} & E_{n 2} & \ldots & E_{n n}
\end{array}\right]\left[\begin{array}{c}
\alpha_{1} \\
\alpha_{2} \\
\cdot \\
\cdot \\
\cdot \\
\alpha_{n}
\end{array}\right]=\left[\begin{array}{c}
E_{11} \\
E_{22} \\
\cdot \\
\cdot \\
\cdot \\
E_{n n}
\end{array}\right]
$$

As $E_{i j}=E_{j i}$ the matrix $\left[E_{i j}\right]$ is symmetric. We should note that $\sum_{i=1}^{n} \alpha_{i}=\mathrm{I}$; so the approximation being considered is free from bias.

It should be easy to prove that, if there are two or more factors with no influence, from the mean value point of view, this system will be indeterminate. In effect, its complete matrix will then have two or more rows linearly dependent. Only after extracting these rows will the system have a unique solution.

Although it has not been conclusively proved we noted that in a large number of practical tests:

I - The factors with little or no influence would systematically induce negative $\alpha$ values.

2 - Eliminating the factors whose $\alpha$ values were negative gives results belonging to the interval $[0, I]$.

In order to study the joint influence we are led to apply once again the previous model, taking now the mean marginal values for the various pairs of parameters.

Let $\mu_{i j}=\mu\left(., \ldots, \theta_{i}, \ldots, \ldots, \theta_{j}, ., \ldots\right)$ with $i \neq j$ and consider $\mu\left(\theta_{T}, \theta_{R}\right)$ approximated by the linear combination

$$
\alpha_{12} \mu_{12}+\ldots+\alpha_{n-1 n} \mu_{n-1 n}
$$

Taking the derivatives in order $\alpha_{i j}$ of an expression similar to (2) and taking $E_{i j k l}$ instead of $E\left[\mu_{i j} \times \mu_{k l}\right]$ with $i \neq j$ and $k \neq l$ we obtain the system 
$\left[\begin{array}{cccc}E_{1212} & E_{1213} & \ldots & E_{12 n-1 n} \\ E_{1312} & E_{1313} & \ldots & E_{13 n-1 n} \\ \cdot & & & \\ \cdot & & \\ E_{n-1 n 12} & E_{n-1 n 13} & \ldots & E_{n-1 n n-1 n}\end{array}\right]\left[\begin{array}{c}\alpha_{12} \\ \alpha_{13} \\ \cdot \\ \cdot \\ \alpha_{n-1 n}\end{array}\right]=\left[\begin{array}{c}E_{1212} \\ E_{1313} \\ \cdot \\ \cdot \\ \cdot \\ E_{n-1 n n-1 n}\end{array}\right]$

This system is similar to the previous one, but could have more equations and unknowns.

The values obtained by solving the system will give us a sufficiently precise idea of the influences attributed to the pairs of parameters.

Should one be interested in establishing a tariff structure, the study of the joint influences and coinfluences seem more important than the actual influences considered one by one (if these exist).

Given the ease of generalisation, the model we have described may have widespread application. We should also note that going from the first to the second system does not necessarily imply an increase in the number of unknowns and equations.

In effect, the number of the different permutations of parameters in the form of combinations taken one by one, two by two, etc., is symmetrical, that is, the first system (obtained by margination in $n$-I parameters) will have as many unknowns and equations as the last, obtained by margination in a single parameter.

\section{Practical Application}

I. In order to test the theoretical models described in this paper we constructed our data, instead of resorting to available statistical information. This enabled us to know the expected behaviour of each parameter from the outset.

We considered 4 parameters, each of them assuming integer values between $I$ and 5 , and we simulated the collective, starting off with mean values obtained by the following equation:

$$
\mu_{i j k l}=7500+1000 \times i+k(200+500 \times j)
$$

In keeping with this deterministic relation we will have:

$\theta_{1}$ - the most influential factor

$\theta_{2}$ - less influential than $\theta_{3}$

$\theta_{2}$ and $\theta_{3}-$ coinfluential

$\theta_{4}$ - non-influential 
It was important to verify the "influence" of the structure function $U(\theta)$ on the model's behaviour. To do so, we carried out two different sets of calculations. In one we included $U(\theta)$ taking different values for $p_{i j k l}$ and in the other we maintained $p_{i j k l}$ constant.

\section{RESULTS}

I.I. Considering the structure function $U(\theta)$

a) Values obtained by the variance model

$I_{1}=I .996 ; I_{2}=.227 ; I_{3}=.552 ; I_{4}=0$

$C I_{12}=.002 ; C I_{13}=.003 ; C I_{14}=0$

$C I_{23}=.04 I ; C I_{24}=0 ; C I_{34}=0$

$C I_{123}=0 \quad ; C I_{124}=0 \quad ; C I_{134}=0 \quad ; C I_{234}=0$

$C I_{1234}=0$

b) Values obtained by the credibility model

\begin{tabular}{ccccc}
\hline N. Eq.coes. & $\alpha_{1}$ & $\alpha_{2}$ & $\alpha_{3}$ & $\alpha_{4}$ \\
\hline 4 & $\mathrm{I}$ & 0 & 1 & -1 \\
3 & .873 & -.373 & .502 & - \\
2 & .797 & - & .206 & - \\
\hline
\end{tabular}

c) Values obtained by the $\chi^{2}$ test applied to the distribution function of each parameter in comparison with the weighted distribution for all the collective.

\begin{tabular}{ccccc}
\hline Valueparam. & $\theta_{1}$ & $\theta_{3}$ & $\theta_{3}$ & $\theta_{4}$ \\
\hline$I$ & 243 & 20.94 & 56.98 & I.37 \\
2 & 79.44 & $I 4.72$ & 25.75 & .63 \\
3 & 1.57 & 2.1 & .87 & 1.86 \\
4 & 54.75 & 7.46 & 17.50 & 1.58 \\
5 & 253.83 & 21.9 & 63.12 & 2.96 \\
\hline Variation & 251.43 & 19.8 & 62.25 & 2.33 \\
\hline
\end{tabular}

From the previous table one can see that the equality of distributions is admissible for the fourth parameter only. 
1.2. Not taking into account the structure function

a) Values obtained by the variance model

$$
\begin{aligned}
& I_{1}=2 ; I_{2}=.22 ; I_{3}=.54 ; I_{4}=0 \\
& C I_{12}=0 ; C I_{13}=0 ; \quad C I_{14}=0 \\
& C I_{23}=.04 ; C I_{24}=0 ; \quad C I_{34}=0 \\
& C I_{123}=0 ; C I_{124}=0 ; C I_{134}=0 ; C I_{234}=0 \\
& C I_{1234}=0
\end{aligned}
$$

b) Values obtained by the credibility model

\begin{tabular}{ccccc}
\hline N. Eq. coet. & $\alpha_{1}$ & $\alpha_{2}$ & $\alpha_{3}$ & $\alpha_{4}$ \\
\hline 4 & $\mathrm{I}$ & $\mathrm{I}$ & $\mathrm{I}$ & -2 \\
3 & .876 & $-.37^{8}$ & .504 & - \\
2 & $.80 \mathrm{I}$ & - & $.20 \mathrm{I}$ & - \\
\hline
\end{tabular}

c) Values obtained by the $\chi^{2}$ test

\begin{tabular}{crrcc}
\hline Value parsm. & \multicolumn{1}{c}{$\theta_{1}$} & $\theta_{2}$ & $\theta_{3}$ & $\theta_{4}$ \\
\hline$I$ & 182.87 & 12.70 & 34.87 & 1.57 \\
2 & 46.82 & 6.51 & 17.76 & 3.24 \\
3 & 2.12 & 1.09 & 3.66 & .3 \\
4 & 63.92 & 4.14 & 4.89 & 1.62 \\
5 & 140.37 & 11.32 & 55.16 & 2.25 \\
\hline Variation & 180.75 & 11.6 & 51.5 & 2.94 \\
\hline
\end{tabular}

I.3 This set of values leads us to conclude that the three models are similar. The variance method, which clearly sets out the influences of the parameters and their respective coinfluences, is nevertheless more sensitive to small variations of the mean values. These properties are not directly found in the other two methods. Nevertheless, if we had applied the complete credibility model, that is, considering the influences of combinations of parameters, we are almost certain that the same conclusions would be reached.

Finally we can see that the results arrived at, on the one hand considering the structure function $U(\theta)$ and on the other hand not taking it into account, are not so different as could have been expected. 


\section{2. $\chi^{2}$ model}

Suppose that we know for each risk $\left(\theta_{T}, \theta_{R}\right)$ the D.F. $G^{\left(\theta_{T}, \theta_{R}\right)}(x)$, at least for the known component $\theta_{T}$ of the risk, and also assume that we know the weighted distribution

$$
G(x)=\int_{T \times \pi} G^{\left(\theta_{T}, \theta_{R}\right)}(x) d U\left(\theta_{T}, \theta_{R}\right)
$$

Intuitively, if a parameter has no influence, all the risks that differ only by the value of that parameter (maintaining the values for the others unaltered) should have the same D.F. Thus, we think it is possible to obtain an idea of the parameter's influence by comparing the D.F. corresponding to each of its values with the weighted D.F. defined over the collective.

As the previous study could lead to such an exaggerated number of D.F.'s we considered it justifiable to simply it, even with loss of precision. In order to do this, we took into account only the marginal D.F. for each value of the parameter (independently of the other parameters).

If we consider the marginal D.F. $G^{\left(\theta_{1}, \cdots\right)}(x), G^{\left(\cdots \theta_{2}, \cdots \cdots\right.}(x)$ and so on, we can compute the values

$$
X^{2}=\sum_{i=1}^{v} \frac{\left(v_{i}^{0}-v_{i}\right)^{2}}{v_{i}}
$$

corresponding to the comparison between the marginal D.F., with $G(x)$. As we all know, if we have the same distribution function, $X^{2}$ will be a $\chi^{2}$ random variable with $N$-I degrees of freedom; but if the two distribution functions are note identical, $X^{2}$ takes on greater values. So, if we compare the $X_{i j}^{2}$ where $i$ is the parameter index and $j$ the value of the parameter, we obtain a set of scaled values which in a certain way measure the influence of each parameter. 